\title{
ON INFLATION-RESTRICTION EXACT SEQUENCES IN GROUP AND AMITSUR COHOMOLOGY
}

\author{
BY \\ A. J. BERKSON AND ALAN McCONNELL( ${ }^{(1)}$
}

1. Introduction. Let $H$ be a normal subgroup of a group $G, M$ a $G$-module; a fundamental theorem of group cohomology [5] states: if $H^{i}(H, M)=0,0<i<n$, then the sequence

$$
\begin{aligned}
0 \longrightarrow H^{n}\left(G / H, M^{H}\right) \stackrel{\lambda}{\longrightarrow} H^{n}(G, M) \stackrel{\zeta}{\longrightarrow} H^{n}(H, M)^{G} \\
\stackrel{t}{\longrightarrow} H^{n+1}\left(G / H, M^{H}\right) \stackrel{\lambda}{\longrightarrow} H^{n+1}(G, M)
\end{aligned}
$$

is exact. In $\$ 2$ of this paper we show (under the assumption that $n>1$ ) that this sequence is embedded in a considerably simpler exact sequence (for the precise statement see Theorem 1) and we are able to say something about the extent of deviation of the two sequences.

Let $C$ be a field, $K \subset F$ extension fields with $[F: C]$ finite; a fundamental theorem of Amitsur cohomology [2], [6], [8] states: the sequence

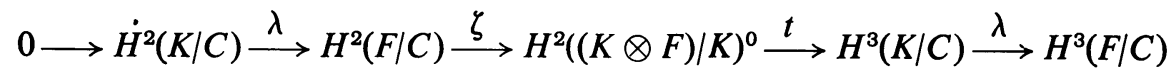

is exact. In $\S 3$ of this paper we show that this sequence is embedded in a considerably simpler exact sequence (for the precise statement see Theorem 2). In $\$ 4$ we discuss the extent of deviation of the two sequences.

Of course these two results are closely related (although neither implies the other) and upon careful analysis it can be seen that their proofs are essentially the same. The group cohomology proof is more elementary in that it does not use spectral sequences; this reflects the fact that the definition of the transgression map $t$ can be stated without mentioning spectral sequences.

Finally, in $\S 5$ we mention an application of these results to the theory of the Brauer group.

2. Group cohomology. For $H, G, M$ as in the introduction, we set $\tilde{M}$ $=\operatorname{Hom}_{H}(G, M)$, the set of functions $f: G \rightarrow M$ such that $f(\tau x)=\tau f(x)$ for $\tau \in H$, $x \in G . \tilde{M}$ is a left $G$-module via ${ }^{\sigma} f(x)=f(x \sigma)$, for $\sigma, x \in G$. We can identify $M$ with the subset $\operatorname{Hom}_{G}(G, M)$ of $\tilde{M}$, making $M$ into a $G$-submodule of $\tilde{M}$. Note that as an $H$-module $\tilde{M}$ is the direct product of $M$ card $(G / H)$ times; this is seen by picking

Received by the editors August 12, 1968.

(1) The second author wishes to acknowledge support of his research by the National Science Foundation under grant GP-6539. 
coset representatives $\left\{\sigma_{i}\right\}$ of $G \bmod H$ and checking that the $H$-module maps $\phi_{i}: \tilde{M} \rightarrow M$, where $\phi_{i}(f)=\sigma_{i}^{-1} f\left(\sigma_{i}\right)$, satisfy the universal property for products. In particular the map $f m f(1)$ yields an $H$-module splitting of the short exact sequence of $G$-modules

$$
0 \longrightarrow M \longrightarrow \tilde{M} \stackrel{\pi}{\longrightarrow} \tilde{M} / M \longrightarrow 0 .
$$

We cite the result sometimes known as Shapiro's Lemma:

Proposition 1. The composite $H^{i}(G, \tilde{M}) \stackrel{\zeta}{\rightarrow} H^{i}(H, \tilde{M}) \rightarrow H^{i}(H, M)$, where $\zeta$ is the restriction homomorphism and the second map is induced by the coefficient homomorphism $\tilde{M} \rightarrow M$ given above, is an isomorphism.

Proof. One is given in [5, p. 114, Corollary].

Proposition 2. $0 \rightarrow M^{H} \rightarrow \tilde{M}^{H} \rightarrow(\tilde{M} / M)^{H} \rightarrow 0$ is exact.

Proof. We know that $0 \rightarrow M^{H} \rightarrow \tilde{M}^{H} \rightarrow(\tilde{M} / M)^{H} \rightarrow H^{1}(H, M)$ is exact and this last group is zero, by the hypothesis $n>1$ made in the introduction.

Proposition 3. $H^{n}\left(G / H, \tilde{M}^{H}\right)=0$ for all $n>0$.

Proof. Let $f \in \tilde{M}^{H}$; then ${ }^{\tau} f(x)=f(x \tau)=f(x)$, for $\tau \in H$. Thus $f$ is constant on cosets of $H$ and we can, and shall, identify $f$ with a map $G / H \rightarrow M$; since $f(\sigma H)$ $=f(\tau \sigma H)=\tau f(\sigma H), f$ takes values in $M^{H}$. As an abelian group, $\tilde{M}^{H}$ is thus isomorphic with Hom $\left(G / H, M^{H}\right)$, and one checks immediately that this is a $G / H$ module isomorphism. $\tilde{M}^{H}$ is thus a co-induced $G / H$-module and hence has zero cohomology [10, p. 120].

COROLlARY. The connecting homomorphisms

$$
\Delta: H^{i}\left(G / H,(\tilde{M} / M)^{H}\right) \rightarrow H^{i+1}\left(G / H, M^{H}\right)
$$

arising from $0 \rightarrow M^{H} \rightarrow \tilde{M}^{H} \rightarrow(\tilde{M} / M)^{H} \rightarrow 0$ are all isomorphisms.

THEOREM 1. There exist maps $m_{1}, m_{3}, m_{4}$ to make the following diagram commutative:

(4)

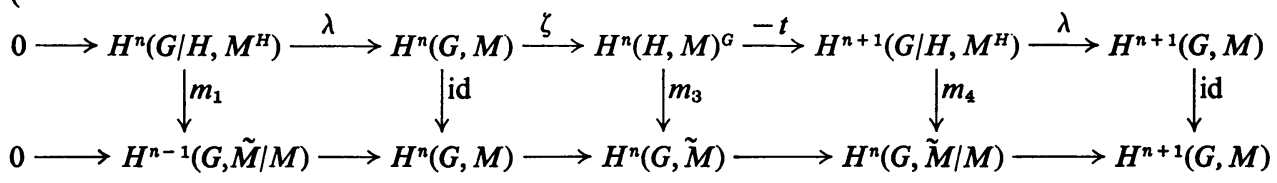

$m_{1}$ is an isomorphism and $m_{3}$ and $m_{4}$ are monomorphisms.

NB: the negative of the transgression map $t$ appears above in order to make the third square commute; we shall show below that with the usual transgression map the third square anticommutes. This is a phenomenon that occurs in the Amitsur cohomology situation also. 
Proof. We define the three nonidentity maps:

$$
\begin{gathered}
m_{1}: H^{n}\left(G / H, M^{H}\right) \cong H^{n-1}\left(G / H,(\tilde{M} / M)^{H}\right) \stackrel{\lambda}{\longrightarrow} H^{n-1}(G, \tilde{M} / M) \\
m_{3}: H^{n}(H, M)^{G} \stackrel{i}{\longrightarrow} H^{n}(H, M) \cong H^{n}(G, \tilde{M})
\end{gathered}
$$

here $\lambda$ is the lift map and $i$ is the injection. We define $m_{4}$ exactly as $m_{1}$ but substituting $n+1$ for $n$.

Note that in the bottom row of (4) $H^{n-1}(G, \tilde{M} / M) \rightarrow H^{n}(G, M)$ is a monomorphism since $H^{n-1}(G, \tilde{M}) \cong H^{n-1}(H, M)=0$.

Square 1. We rewrite it as follows:

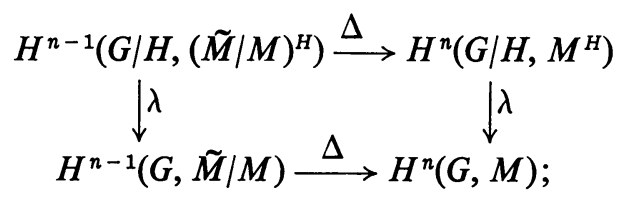

and this, by well-known properties of the lift map, commutes. The proof for Square 4 is exactly the same.

Square 2. We rewrite it as follows:

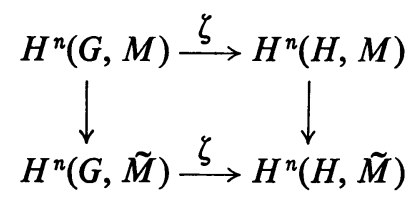

where the vertical maps are induced by $M \rightarrow \tilde{M}$; note that the Shapiro isomorphism runs from lower left to upper right. This diagram commutes, by well-known properties of the restriction map.

Square 3. We recall the definition of $t: H^{n}(H, M)^{G} \rightarrow H^{n+1}\left(G / H, M^{H}\right)$ [5]. Let $\alpha: H^{n} \rightarrow M$ be a cocycle representing a $G$-fixed cohomology class of $H^{n}(H, M)$ (NB: All our cocycles and cochains will be normalized; i.e., will take value $0 \in M$ on any $n$-tuple of group elements containing $1 \in G$ as an entry.) We know then that $\alpha$ is transgressive; i.e., there exists a cochain $A: G^{n} \rightarrow M$ such that $A$ restricted to $H^{n}$ equals $\alpha$ and $\delta A: G^{n+1} \rightarrow M$ is constant on cosets and takes values in $M^{H}$. Let $\beta:(G / H)^{n+1} \rightarrow M^{H}$ be the function $\delta A$ induces; $\beta$ is a cocycle, and by definition of $t, t(\operatorname{cl} \alpha)=\operatorname{cl} \beta$.

Pick $\bar{\gamma}:(G / H)^{n} \rightarrow(\tilde{M} / M)^{H}$ a cocycle representing the inverse image of $\operatorname{cl} \beta$ under the isomorphism $\Delta: H^{n}\left(G / H,(\tilde{M} / M)^{H}\right) \rightarrow H^{n+1}\left(G / H, M^{H}\right)$; we can find $\gamma:(G / H)^{n}$ $\rightarrow \tilde{M}^{H}$, a "lift" of $\bar{\gamma}$, such that $\delta \gamma=\beta$ as maps $(G / H)^{n+1} \rightarrow M^{H}$. Let $\gamma_{0}: G^{n} \rightarrow \tilde{M}^{H}$ be the "pullback" of $\gamma$.

Consider $\gamma_{0}-A: G^{n} \rightarrow \tilde{M}$; since both $\delta \gamma_{0}$ and $\delta A$ are constant on cosets and are equal to $\beta$ when "collapsed" to maps $(G / H)^{n+1} \rightarrow M^{H}$, we see that $\delta \gamma_{0}=\delta A$ as maps $G^{n+1} \rightarrow \tilde{M}$ and hence $\gamma_{0}-A$ is a cocycle representing an element of $H^{n}(G, \tilde{M})$. Since $A$ takes values in $M \subset \tilde{M}, \mathrm{cl}\left(\gamma_{0}-A\right)$ maps to the same element in $H^{n}(G, \tilde{M} / M)$ 
as does $\mathrm{cl}(\bar{\gamma})$. Since $\gamma_{0}$ is the "pull back" of $\gamma:(G / H)^{n} \rightarrow \tilde{M}$, and since all cochains are normalized, $\gamma_{0}-A$ restricts to the same function as $-A$ does on $H^{n}$, namely $-\alpha$. This proves the anticommutativity of the third square.

Finally, we note that $m_{3}$ is a monomorphism and hence $m_{4}$ is too, by the Five Lemma. Furthermore, $m_{1}$ must be an isomorphism, since the kernels of the horizontal maps in the second square are the same. This concludes the proof of Theorem 1 .

To what extent do these two sequences deviate? Letting $M_{i}=$ coker $m_{i}, i=3,4$, we note that there is a map $M_{3} \rightarrow M_{4}$, and a routine diagram chase shows that it is a monomorphism. We have already noted that $\tilde{M}$ is the direct product of copies of $M$ as an $H$-module. Since the functors $H^{i}(H,-)$ preserve direct products, we have $H^{i}(H, \tilde{M})=\Pi_{k} H^{i}(H, M)_{k}=0$ for $0<i<n$. Since $0 \rightarrow M \rightarrow \tilde{M} \rightarrow \tilde{M} / M \rightarrow 0$ splits as $H$-module sequence, it is clear that $H^{i}(H, \tilde{M} / M)=0$ for $0<i<n$. This means that we may apply the exact sequence (1), substituting $\tilde{M} / M$ for $M$, and we see that $M_{4}$ is a subgroup of $H^{n}(H, \tilde{M} / M)^{G}$. Certainly if this last group is zero, then our two sequences are identical.

Corollary. An element $x \in H^{n}(H, M)$ is $G$-fixed, i.e., in $H^{n}(H, M)^{G}$, if and only if it is taken to zero under the composite map

$$
H^{n}(H, M) \cong H^{n}(G, \tilde{M}) \rightarrow H^{n}(G, \tilde{M} / M) \rightarrow H^{n}(H, \tilde{M} / M) .
$$

3. Amitsur cohomology. (For the basic definitions and theorems of Amitsur cohomology, see [1], [3] and [7].) All tensor products shall be over $C$, unless otherwise noted; we set $F^{n}=F \otimes \cdots \otimes F$ ( $n$ times). We recall the existence of "face" maps $\varepsilon_{i}: F^{n} \rightarrow F^{n+1}(i=1, \ldots, n+1)$ and "degeneracy" maps $s_{i}: F^{n+1} \rightarrow F^{n}$ $(i=1, \ldots, n+1)$. If $T$ is a covariant functor from the category of commutative $C$-algebras to that of abelian groups, we can define a cochain complex $\mathscr{C}(F / C, T)$, $\cdots \rightarrow T\left(F^{n-1}\right) \rightarrow T\left(F^{n}\right) \rightarrow T\left(F^{n+1}\right) \rightarrow \cdots$, where the differentiation $d: T\left(F^{n}\right)$ $\rightarrow T\left(F^{n+1}\right)$ is given by $\sum_{i=0}^{n} T\left(\varepsilon_{i}\right)^{(-1)^{t}}$. The cohomology groups of this complex are denoted, following [3], by $H^{n}(F / C, T)(n \geqq 0)$. Among the functors we shall be considering are the "units" functor $U$, which assigns to every commutative $C$ algebra $X$ its group of units $U(X)=X^{*}$, the functor $U K$, where $U K(X)=(K \otimes X)^{*}$, and the quotient functor of these two functors $Q$, where $Q(X)=(K \otimes X)^{*} /(1 \otimes X)^{*}$. In [1] and [7] $H^{n}(F / C, U)$ is written simply $H^{n}(F / C)$, a practice we shall follow. The symbols $\lambda$ and $\zeta$ will stand in a general way for the lift and restriction mappings of Amitsur cohomology; they are induced by $i: K \rightarrow F$ and $l: F \rightarrow K \otimes F(l(f)$ $=1 \otimes f)($ see $[2])$.

We first record a result related to Proposition 3 of $\$ 2$.

Proposition 1. $H^{n}(K / C, U K)=0$ for all $n \geqq 0$.

Proof. See [2, Theorem 2.9]. The idea is: if one tensors the complex $K \rightarrow K^{2}$ $\rightarrow \cdots$ with $K$ one can collapse a factor and easily get a contracting homotopy. 
COROllary. The connecting homomorphism: $\Delta: H^{n-1}(K / C, Q) \rightarrow H^{n}(K / C)$ arising from the exact sequence of complexes

$$
1 \rightarrow \mathscr{C}(K / C, U) \rightarrow \mathscr{C}(K / C, U K) \rightarrow \mathscr{C}(K / C, Q) \rightarrow 1
$$

is an isomorphism for each $n \geqq 1$.

Now consider the short exact sequence of complexes $1 \rightarrow \mathscr{C}(F / C, U) \stackrel{\iota}{\rightarrow}$ $\mathscr{C}(F / C, U K) \stackrel{\pi}{\rightarrow} \mathscr{C}(F / C, Q) \rightarrow 1$; this gives rise to a long exact sequence of cohomology:

$$
\begin{aligned}
\cdots \longrightarrow H^{n-1}(F / C, Q) \stackrel{\Delta}{\longrightarrow} & H^{n}(F / C) \stackrel{l^{*}}{\longrightarrow} H^{n}(F / C, U K) \\
& \stackrel{\pi^{*}}{\longrightarrow} H^{n}(F / C, Q) \longrightarrow H^{n+1}(F / C) \longrightarrow \cdots
\end{aligned}
$$

THEOREM 2. There exist maps $m_{1}, m_{3}, m_{4}$ to make the following diagram commutative:

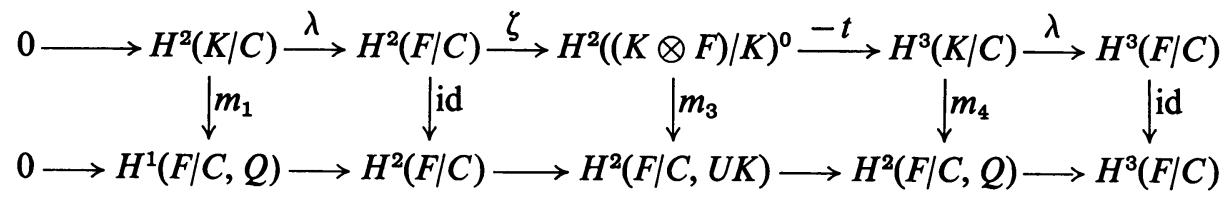

$m_{1}$ is an isomorphism and $m_{3}$ and $m_{4}$ are monomorphisms.

NB: See the note after the statement of Theorem 1.

Proof. We define the three nonidentity maps. There are homomorphisms $\left(K \otimes K^{n}\right)^{*} \rightarrow\left(K \otimes F^{n}\right)^{*}$ and $\left(1 \otimes K^{n}\right)^{*} \rightarrow\left(1 \otimes F^{n}\right)^{*}$; these induce a map of complexes $\mathscr{C}(K / C, Q) \rightarrow \mathscr{C}(F / C, Q)$ and hence a map of cohomology $\lambda: H^{n}(K / C, Q)$ $\rightarrow H^{n}(F / C, Q)$. Using the Corollary to Proposition 1, we define $m_{1}: H^{2}(K / C)$ $\cong H^{1}(K / C, Q) \stackrel{\lambda}{\rightarrow} H^{1}(F / C, Q)$, and $m_{4}$ is the same, one dimension higher. Identifying $K \otimes F^{n}$ with $(K \otimes F) \otimes_{K} \cdots \otimes_{K}(K \otimes F)\left(n\right.$ times) we see that $H^{n}(F / C, U K)$ and $H^{n}((K \otimes F) / K)$ can be identified; $m_{3}$ is simply the embedding map.

Note that in the bottom row of $(5) H^{1}(F / C, Q) \rightarrow H^{2}(F / C)$ is a monomorphism, since $H^{1}(F / C, U K)=0$ [2, Theorem 3.8].

For the rest of this proof we shall write simply $F, K \otimes F^{2}$, etc., instead of $F^{*}$, $\left(K \otimes F^{2}\right)^{*}$, as all groups considered will be groups of units.

Square 1. We rewrite it as follows:

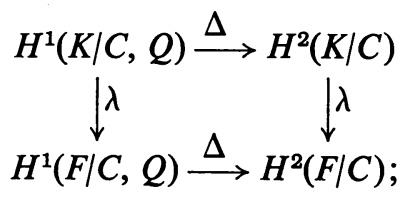

that this diagram of Amitsur cohomology commutes is well known, and a routine computation to check. The proof for Square 4 is the same. 
Square 2. We have noted that the two horizontal maps are both induced by $l: F \rightarrow K \otimes F$ and this, together with the definition of $m_{3}$ as the inclusion mapping, shows that Square 2 commutes.

Square 3. We embark upon an analysis of the "transgression" map

$$
t: H^{2}((K \otimes F) / K)^{0} \rightarrow H^{3}(K / C) ;
$$

in doing so we use the results and notation of [6]. (For notation and theory of spectral sequences, see [9].) In [6] the double complex $E_{0}^{m, n}=K^{m+1} \otimes F^{n+1}$ (always multiplicative groups, as noted) was considered, together with the "first spectral sequence"

$$
E_{2}^{m, n}=H^{m}\left(H^{n}(E)\right) \underset{m}{\Rightarrow} H(\mathrm{Tot}) .
$$

LEMMA 1 [6, LEMMA 1.3]. Let $E_{2}^{m, n} \underset{m}{\Rightarrow} H$ (Tot) be a spectral sequence such that $E_{2}^{m, n}=0$ if either $m$ or $n$ is less than 0 and such that $E_{2}^{1,1}=E_{2}^{2,1}=0$; then there are maps to make

$$
E_{2}^{0,1} \rightarrow E_{2}^{2,0} \rightarrow H^{2}(\mathrm{Tot}) \rightarrow E_{2}^{0,2} \rightarrow E_{2}^{3,0} \rightarrow H^{3}(\mathrm{Tot})
$$

exact.

(This is an elementary spectral sequence result. In [6] it was further shown that $E_{2}^{0,1}, E_{2}^{2,0}, H^{2}, E_{2}^{0,2}, E_{2}^{3,0}$, and $H^{3}$ can be identified with $0, H^{2}(K / C), H^{2}(F / C)$, $H^{2}((K \otimes F) / K)^{0}, H^{3}(K / C)$ and $H^{3}(F / C)$ respectively, and (2) follows.)

What is $t: E_{2}^{0,2} \rightarrow E_{2}^{3,0}$ ? We first note that $E_{2}^{0,2}=E_{3}^{0,2}$; indeed, there are no boundaries in $E_{2}^{0,2}$ since $\left\{E_{2}^{m, n}\right\}$ is a "first quadrant" bigraded module, and every element of $E_{2}^{0,2}$ is a cycle since $d_{2}$ maps $E_{2}^{0,2}$ to $E_{2}^{2,1}=0$. A dual argument, using the hypothesis that $E_{2}^{1,1}=0$, shows that $E_{2}^{3,0}=E_{3}^{3,0}$. Now an examination of the proof of the above lemma reveals that $t$ is simply the composite $E_{2}^{0,2} \cong E_{3}^{0,2} \rightarrow E_{3}^{3,0} \cong E_{2}^{3,0}$.

We compute $d_{3}$. By definition

$$
d_{3}: E_{3}^{0,2}=Z_{3}^{0,2} /\left(Z_{2}^{1,1}+B_{2}^{0,2}\right) \rightarrow E_{3}^{3,0}=Z_{3}^{3,0} / B_{2}^{3,0}
$$

is defined on an element $\bar{x} \in E_{3}^{0,2}, x \in Z_{3}^{0,2}$, by taking its total differential $d x \in Z_{3}^{3,0}$ and then its class modulo $B_{2}^{3,0}$. Now

$$
x=\left(x^{0,2}, x^{1,1}, x^{2,0}\right) \in Z_{3}^{3,0} \subset E_{0}^{0,2} \oplus E_{0}^{1,1} \oplus E_{0}^{2,0}
$$

is, by definition of $Z_{3}^{0,2}$, dropped three levels down in the filtration by $d$. From the definition of the total differential $d$ in a double complex we see that the following relations hold (' $d$ denotes horizontal differential, " $d$ the vertical):

(i) $" d x^{0,2}=0$,

(ii) ' $d x^{0,2}+" d x^{1,1}=0$,

(iii) ${ }^{\prime} d x^{1,1}+{ }^{\prime \prime} d x^{2,0}=0$,

(iv) $d x={ }^{\prime} d x^{2,0} \in Z_{3}^{3,0}$.

We apply this to our case, where $E_{0}^{m, n}$ is just (the group of units of) $K^{m+1} \otimes F^{n+1}$. Let $\alpha \in K \otimes F^{3}$ represent an element of $H^{2}((K \otimes F) / K)^{0}$. (We recall that

$$
H^{2}((K \otimes F) / K)^{0}
$$


is, by definition, the set of elements $z \in H^{2}((K \otimes F) / K)$ for which $\varepsilon_{1}^{*}(z)=\varepsilon_{2}^{*}(z)$, where $\varepsilon_{i}^{*}: H^{2}((K \otimes F) / K) \rightarrow H^{2}\left(\left(K^{2} \otimes F\right) / K^{2}\right)$ are the maps induced by $\varepsilon_{i}: K \rightarrow K^{2}$. I.e., $H^{2}((K \otimes F) / K)^{0}=\operatorname{Ker}\left(d_{1}: E_{1}^{0,2} \rightarrow E_{1}^{1,2}\right)=E_{2}^{0,2}$, as previously asserted.) We have

(i)' " $d \alpha=\left(\varepsilon_{2} \varepsilon_{4} / \varepsilon_{3} \varepsilon_{5}\right) \alpha=1$,

(ii)' there exists $q \in K^{2} \otimes F^{2}$ such that $\left(\varepsilon_{1} / \varepsilon_{2}\right) \alpha \cdot\left(\varepsilon_{4} / \varepsilon_{3} \varepsilon_{5}\right) q=1$,

(iii)' there exists $u \in K^{3} \otimes F$ such that $\left(\varepsilon_{1} \varepsilon_{3} / \varepsilon_{2}\right) q \cdot\left(\varepsilon_{4} / \varepsilon_{5}\right) u=1$,

(iv) $d(\alpha, q, u)=^{\prime} d u=\left(\varepsilon_{1} \varepsilon_{3} / \varepsilon_{2} \varepsilon_{4}\right) u$ is an element $v \in K^{4} \otimes F$.

$\left(\left(\varepsilon_{2} \varepsilon_{4} / \varepsilon_{3} \varepsilon_{5}\right) \alpha\right.$ means $\varepsilon_{2}(\alpha) \cdot \varepsilon_{3}(\alpha)^{-1} \cdot \varepsilon_{4}(\alpha) \cdot \varepsilon_{5}(\alpha)^{-1}$, etc). By the anticommutativity of the double complex we have $\left(\varepsilon_{5} / \varepsilon_{6}\right) v=" d v=1$, and hence $v$ is actually in $K^{4} \otimes 1$ $\subset K^{4} \otimes F$, since the sequence $1 \rightarrow K^{4} \rightarrow K^{4} \otimes F \rightarrow K^{4} \otimes F^{2}$ is exact. We write $v=\beta \otimes 1, \beta \in K^{4}$, and it is clear that $\operatorname{cl}(\beta) \in H^{3}(K / C)$ is $t(\mathrm{cl} \alpha)$.

We note three facts about $\beta$. First and most important, it is a cocycle; i.e.,

$$
\left(\varepsilon_{1} \varepsilon_{3} \varepsilon_{5} / \varepsilon_{2} \varepsilon_{4}\right) \beta=1 \text {. }
$$

Second, since $\mathrm{cl} \beta$ goes to zero under $\lambda: H^{3}(K / C) \rightarrow H^{3}(F / C)$-this is because (2) is exact-we can find an element $\theta \in F^{3}$ such that

$$
\left(\varepsilon_{1} \varepsilon_{3} / \varepsilon_{2} \varepsilon_{4}\right) \theta=\beta .
$$

Third, $\beta \in K^{4}$ is an element of $K \otimes F^{3}, K^{2} \otimes F^{2}$ and $K^{3} \otimes F$. We exploit these three facts, together with our freedom in choosing $q$ and $u$, to prove

LEMMA 2. $\operatorname{cl}\left(\beta^{-1}(1 \otimes \theta)\right) \in H^{2}((K \otimes F) / K)^{0}$ and is in the preimage of $t(\mathrm{cl} \alpha)$ $=\operatorname{cl} \beta \in H^{3}(K / C)$.

Proof. $\left(\varepsilon_{2} \varepsilon_{4} / \varepsilon_{3} \varepsilon_{5}\right)(1 \otimes \theta)=1 \otimes \beta=\varepsilon_{1} \beta=\left(\varepsilon_{2} \varepsilon_{4} / \varepsilon_{3} \varepsilon_{5}\right) \beta$, using (6) and (7). This proves that $\beta^{-1}(1 \otimes \theta)$ is a cocycle, representing an element of $H^{2}((K \otimes F) / K)$.

Because of $(6),\left(\varepsilon_{1} / \varepsilon_{2}\right) \beta^{-1}(1 \otimes \theta)=\left(\varepsilon_{1} / \varepsilon_{2}\right) \beta^{-1}=\left(\varepsilon_{3} \varepsilon_{5} / \varepsilon_{4}\right) \beta$; hence

$$
\operatorname{cl}\left(\beta^{-1}(1 \otimes \theta)\right) \in H^{2}((K \otimes F) / K)^{0} .
$$

Again because of (6) $\left(\varepsilon_{1} \varepsilon_{3} / \varepsilon_{2}\right) \beta \cdot\left(\varepsilon_{4} / \varepsilon_{5}\right) \beta^{-1}=1$. And yet again because of (6), $\left(\varepsilon_{1} \varepsilon_{3} / \varepsilon_{2} \varepsilon_{4}\right) \beta^{-1}=\varepsilon_{5} \beta=\beta \otimes 1 \in K^{4} \otimes 1 \subset K^{4} \otimes F$. This means, in the notation of (i)'-(iv)', that we can set $q=\beta$ and $u=\beta^{-1}$ when we are computing the image of $\beta^{-1}(1 \otimes \theta)$ under $t$, and we find that $t\left(\operatorname{cl}\left(\beta^{-1}(1 \otimes \theta)\right)\right)$ is indeed $\mathrm{cl} \beta$, which proves the lemma.

We now consider the connecting homomorphism $\Delta: H^{2}(K / C, Q) \rightarrow H^{3}(K / C)$, which is an isomorphism by the corollary to Proposition 1 . Let the unique preimage of $\operatorname{cl} \beta \in H^{3}(K / C)$ be $\operatorname{cl} \tilde{\gamma}, \tilde{\gamma} \in\left(K \otimes K^{3}\right) /\left(1 \otimes K^{3}\right)$. Recalling the definition of $\Delta$, we see that there exists $\gamma \in K \otimes K^{3}$ representing $\tilde{\gamma}$ such that $\left(\varepsilon_{2} \varepsilon_{4} / \varepsilon_{3} \varepsilon_{5}\right) \gamma$ $=1 \otimes \beta \in 1 \otimes K^{3}$. But $1 \otimes \beta=\varepsilon_{1} \beta$, and by the ubiquitous (6), which says that $\left(\varepsilon_{2} \varepsilon_{4} / \varepsilon_{3} \varepsilon_{5}\right) \beta=\varepsilon_{1} \beta$, we see that $\beta \in K^{4}=K \otimes K^{3}$ is a representative of

$$
\tilde{\gamma} \in\left(K \otimes K^{3}\right) /\left(1 \otimes K^{3}\right)
$$


With these facts established we can now show that the third square commutes. Since $t(\operatorname{cl} \alpha)=t\left(\operatorname{cl} \beta^{-1}(1 \otimes \theta)\right)$ and $\operatorname{Ker} t \cong \operatorname{Ker} \pi^{*}$, we conclude that $\pi^{*}(\operatorname{cl} \alpha)$ $=\pi^{*}\left(\operatorname{cl} \beta^{-1}(1 \otimes \theta)\right)$. But $\pi^{*}$ is induced by $\pi: K \otimes F^{3} \rightarrow\left(K \otimes F^{3}\right) /\left(1 \otimes F^{3}\right)$, and hence $\pi^{*}\left(\operatorname{cl} \beta^{-1}(1 \otimes \theta)\right)=\operatorname{cl}\left(\widetilde{\beta}^{-1}\right)$, where $\beta^{-1}=\pi \widetilde{\beta}^{-1} \in\left(K \otimes F^{3}\right) /\left(1 \otimes F^{3}\right)$.

We have seen that $\beta \in K^{4}=K \otimes K^{3} \subset K \otimes F^{3}$ is a representative of

$$
\tilde{\gamma} \in\left(K \otimes K^{3}\right) /\left(1 \otimes K^{3}\right),
$$

where $\operatorname{cl}(\tilde{\gamma})=\Delta^{-1}(\operatorname{cl} \beta)=\Delta^{-1}(t(\operatorname{cl} \alpha))$. Thus the lift map $\lambda$, induced by

$$
\left(K \otimes K^{3}\right) /\left(1 \otimes K^{3}\right) \rightarrow\left(K \otimes F^{3}\right) /\left(1 \otimes F^{3}\right),
$$

takes $\operatorname{cl}(\tilde{\gamma})$ to $\operatorname{cl}(\tilde{\beta}) \in H^{2}(F / C, Q)$. We thus have: $\lambda \Delta^{-1} t(\operatorname{cl} \alpha)=\operatorname{cl} \tilde{\beta} \in H^{2}(F / C, Q)$, while $\pi^{*}(\operatorname{cl} \alpha)=\operatorname{cl} \tilde{\beta}^{-1}$. From this it is clear (note the $-t$ !) that the third square commutes.

Finally, we note that $m_{3}$ is a monomorphism, and hence $m_{4}$ is too, by the Five Lemma. Furthermore $m_{1}$ must be an isomorphism, since the kernels of the horizontal maps in the second square are the same. This concludes the proof of Theorem 2.

REMARK. The isomorphism of $H^{2}(K / C)$ with $H^{1}(F / C, Q)$ is a known result; see $[1],[2]$.

4. The "fundamental exact sequence" in Amitsur cohomology. To measure the deviation of the two sequences of (5) it is necessary to produce a "lift-restriction" exact sequence of which $0 \rightarrow H^{2}(K / C, Q) \rightarrow H^{2}(F / C, Q)$ is the beginning. This requires slightly more work than in the group cohomology case, since here the dimension-shifting techniques are not so readily available, but the requisite sequence is easily exhibited by aping the arguments of [6, Lemma 1.3 and surroundings].

For $m>0$, let the functor $Q_{m}$ be defined by

$$
Q_{m}(X)=\left(K \otimes\left(K^{m} \otimes X\right)\right)^{*} /\left(1 \otimes\left(K^{m} \otimes X\right)\right)^{*} ;
$$

$Q_{m}$ is thus the quotient functor of $U K^{m+1}$ by $U K^{m}$.

Proposition 1. The complex $\mathscr{C}\left(F / C, U K^{m}\right)$ is a direct summand of the complex $\mathscr{C}\left(F / C, U K^{m+1}\right)$.

Proof. For each $n \geqq 0$, the map $x \otimes y m 1 \otimes x \otimes y\left(x \in K^{m}, y \in F^{n+1}\right)$ is an injection, and a splitting is the map $z \otimes y m z^{\prime} \otimes y$ where $z^{\prime}=s_{1}(z)$. Both these maps are chain maps.

COROLlary. For each $m>0$ and $n \geqq 0$, the following sequence is split exact:

$$
0 \rightarrow H^{n}\left(F / C, U K^{m}\right) \rightarrow H^{n}\left(F / C, U K^{m+1}\right) \rightarrow H^{n}\left(F / C, Q_{m}\right) \rightarrow 0 .
$$

Now consider the double complex

$$
E_{0}^{m, n}=Q\left(K^{m+1} \otimes F^{n+1}\right)=\left(K \otimes K^{m+1} \otimes F^{n+1}\right)^{*} /\left(1 \otimes K^{m+1} \otimes F^{n+1}\right)^{*}
$$


As usual we denote the first spectral sequence arising from this double complex by ' $E$, the second by " $E$.

Proposition 2. ' $E_{1}^{m, 1}=0$ for $m \geqq 0$.

Proof. ' $E_{1}^{m, 1}=H^{1}\left(F / C, Q_{m+1}\right)$. Now $H^{1}\left(F / C, U K^{m+1}\right)=H^{1}\left(F / C, U K^{m+2}\right)=0$ by the elementary properties of fields, and the corollary of Proposition 1 gives the result.

We can now quote Lemma $1, \S 3$, to deduce that there is an exact sequence

$$
0 \rightarrow{ }^{\prime} E_{2}^{2,0} \rightarrow H^{2} \text { (Tot) } \rightarrow^{\prime} E_{2}^{0,2} \rightarrow^{\prime} E_{2}^{3,0} \rightarrow H^{3}(\text { Tot })
$$

and it remains only to identify these groups of our spectral sequence.

Proposition 3. ' $E_{2}^{m, 0}=H^{m}(K / C, Q)$.

Proof. By [6, Lemma 1.1] the sequence

$$
1 \rightarrow\left(K^{m}\right)^{*} \rightarrow\left(K^{m} \otimes F\right)^{*} \rightarrow\left(K^{m} \otimes F^{2}\right)^{*}
$$

is exact for all $m \geqq 0$. This says simply that

$$
\left(K^{m}\right)^{*}=H^{0}\left(\left(K^{m} \otimes F\right) / K^{m}\right)=H^{0}\left(F / C, U K^{m}\right) .
$$

From the corollary of Proposition 1 we deduce that

$$
{ }^{\prime} E_{1}^{m, 0}=\left(K \otimes K^{m+1}\right)^{*} /\left(1 \otimes K^{m+1}\right)^{*},
$$

and hence the result.

Proposition 4. " $E_{1}^{m, n}=0$ for $m>0$ and $" E_{1}^{0, n}=Q\left(F^{n+1}\right)$.

Proof. For fixed $n \geqq 0$, consider the complex

$$
\left(K \otimes K \otimes F^{n+1}\right)^{*} \rightarrow\left(K \otimes K^{2} \otimes F^{n+1}\right)^{*} \rightarrow\left(K \otimes K^{3} \otimes F^{n+1}\right)^{*} \rightarrow \cdots
$$

and its subcomplex

$$
\left(1 \otimes K \otimes F^{n+1}\right)^{*} \rightarrow\left(1 \otimes K^{2} \otimes F^{n+1}\right)^{*} \rightarrow\left(1 \otimes K^{3} \otimes F^{n+1}\right)^{*} \rightarrow \cdots ;
$$

the quotient complex is $E_{0}^{0, n} \rightarrow E_{0}^{1, n} \rightarrow E_{0}^{2, n} \rightarrow \cdots$. It is proved in [6, Lemma 1.2], following [2], that the cohomology of the first two complexes is zero in dimensions greater than zero, and that the zeroth cohomology groups are $\left(K \otimes 1 \otimes F^{n+1}\right)^{*}$ $\cong\left(K \otimes F^{n+1}\right)^{*}$ and $\left(1 \otimes 1 \otimes F^{n+1}\right) \cong\left(1 \otimes F^{n+1}\right)^{*}$ respectively. Hence the result.

Corollary. $H^{n}($ Tot $)=" E_{2}^{0, n}=H^{n}(F / C, Q)$.

Proof. As in the proof of [6, Lemma 1.2], this is a standard result about "collapsing" spectral sequences.

Finally, we consider ' $E_{2}^{0,2}$. We have ' $E_{1}^{0,2}=H^{2}\left(F / C, Q_{1}\right)$, and hence

$$
{ }^{\prime} E_{2}^{0,2}=H^{2}\left(F / C, Q_{1}\right)^{0}=\left\{x \in H^{2}\left(F / C, T_{1}\right): Q\left(\varepsilon_{1}\right)^{*}(x)=Q\left(\varepsilon_{2}\right)^{*}(x)\right\}
$$


where the $Q\left(\varepsilon_{i}\right)^{*}$ are the maps on cohomology induced by

$$
Q\left(\varepsilon_{i}\right): Q\left(K \otimes F^{3}\right) \rightarrow Q\left(K^{2} \otimes F^{3}\right)(i=1,2) .
$$

THEOREM 3. The following sequence is exact:

$$
0 \rightarrow H^{2}(K / C, Q) \stackrel{\lambda}{\rightarrow} H^{2}(F / C, Q) \stackrel{\zeta}{\rightarrow} H^{2}\left(F / C, Q_{1}\right)^{0} \rightarrow H^{3}(K / C, Q) \stackrel{\lambda}{\rightarrow} H^{3}(F / C, Q) .
$$

Proof. After the foregoing series of propositions, it remains only to remark that the maps in question are indeed induced by $i: K \rightarrow F$ and $l: F \rightarrow K \otimes F$; this can be seen exactly as in [6, Proposition 1.6] where the analogous claim is made for the spectral sequence involving the functor $U$ instead of our $Q$.

Corollary. An element $x \in H^{2}((K \otimes F) / K)$ is transgressive, i.e. in

$$
H^{2}((K \otimes F) / K)^{0} \text {, }
$$

if and only if it is taken to zero under the composite map

$$
H^{2}((K \otimes F) / K)=H^{2}(F / C, U K) \rightarrow H^{2}(F / C, Q) \rightarrow H^{2}\left(F / C, Q_{1}\right) .
$$

5. The Brauer group. Let $K$ now be a finite Galois extension of $C$ and $F$ a finite Galois extension of both $K$ and $C$; set $G=\mathscr{G}(F / C), H=\mathscr{G}(F / K) \subset G$, and hence $G / H=\mathscr{G}(K / C)$. The multiplicative group $F^{*}$ is a $G$-module (denote the action of $\sigma \in G$ on $f \in F$ by $\left.{ }^{\sigma} f\right)$ and we make $(K \otimes F)^{*}$ into a $G$-module by letting $G$ act on the second factor (not the diagonal action).

Proposition 1. There exists a function $\psi:(K \otimes F)^{*} \rightarrow \operatorname{Hom}_{H}\left(G, F^{*}\right)$ which is a G-module isomorphism, and the following diagram is commutative:

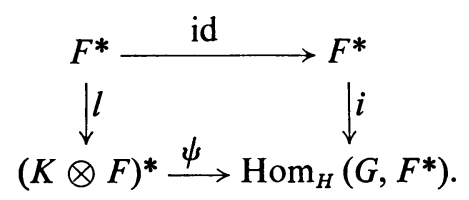

Proof. Define $\quad \psi: K \otimes F \rightarrow \operatorname{Hom}(G, F)$ by $\psi\left(\sum_{i} k_{i} \otimes f_{i}\right)(\sigma)=\sum_{i} k_{i}^{\sigma} f_{i}$. One verifies the following easily:

(i) $\psi\left(\sum_{i} k_{i} \otimes f_{i}\right): G \rightarrow F$ is in $\operatorname{Hom}_{H}(G, F)$,

(ii) $\psi$ is a $G$-module map,

(iii) $\psi$ is a $C$-algebra map.

Now $\psi$ is a monomorphism, by the linear dependence of automorphisms of $G$, and an easy dimension-counting argument shows that $\psi$ is therefore surjective also. It remains only to remark that the group of units of the $C$-algebra $\operatorname{Hom}_{H}(G, F)$ is exactly the group $\operatorname{Hom}_{H}\left(G, F^{*}\right)$; the commutativity of the diagram is immediate.

We can now rephrase the corollary of Theorem 1 as follows:

Proposition 2. An element $x \in H^{2}\left(H, F^{*}\right)$ is $G$-fixed, i.e., in $H^{2}\left(H, F^{*}\right)^{G}$, if and only if it goes to zero under the composite

$$
H^{2}\left(H, F^{*}\right) \cong H^{2}\left(G,(K \otimes F)^{*}\right) \rightarrow H^{2}\left(G, \frac{(K \otimes F)^{*}}{(1 \otimes F)^{*}}\right) \rightarrow H^{2}\left(H, \frac{(K \otimes F)^{*}}{(1 \otimes F)^{*}}\right)
$$


Finally, we recall the interpretation of $H^{2}\left(H, F^{*}\right)^{G}$ as a subgroup of $H^{2}\left(H, F^{*}\right)$ $\cong B(F / K)$, the Brauer group of all algebra classes with center $K$ that are split by $F$ : namely, $x \in H^{2}\left(H, F^{*}\right)$ is in $H^{2}\left(H, F^{*}\right)^{G}$ if and only if its corresponding algebra class is $G$-normal, i.e., every $C$-automorphism of the center $K$ of the algebra $A \in x$ can be extended to an automorphism of the full algebra $A$ [4], [5], [11]. Proposition 2 thus yields a criterion for $G$-normality of an algebra.

\section{REFERENCES}

1. S. A. Amitsur, Simple algebras and cohomology groups of arbitrary fields, Trans. Amer. Math. Soc. 90 (1959), 73-112.

2. _- Homology groups and double complexes for arbitrary fields, J. Math. Soc. Japan, 14 (1962), 1-25.

3. S. U. Chase, D. K. Harrison and Alex Rosenberg, Galois theory and cohomology of commutative rings, Mem. Amer. Math. Soc. No. 52 (1965).

4. S. Eilenberg and S. McLane, Cohomology and Galois theory. I, Normality of algebras and Teichmüller's cocycle, Trans. Amer. Math. Soc. 64 (1948), 1-20.

5. G. Hochschild and J.-P. Serre, Cohomology of group extensions, Trans. Amer. Math. Soc. 74 (1953), 110-134.

6. Bodo Paregis and Alex Rosenberg, Addendum to "Amitsur's complex for inseparable fields", Osaka J. Math. 1 (1964), 33-44.

7. A. Rosenberg and D. Zelinsky, On Amitsur's complex, Trans. Amer. Math. Soc. 97 (1960), 327-356.

8. —-, Amitsur's complex for inseparable fields, Osaka Math. J. 14 (1962), 219-240.

9. J.-P. Serre, Homologie singulière des espaces fibrés, Ann. of Math. 54 (1951), 425-505.

10. - Corps locaux, Hermann, Paris, 1962.

11. O. Teichmüller, Uber die sogenannte nichtkommutative Galoissche Theorie und die Relation $\xi_{\lambda, u, v} \xi_{\lambda, u v, \pi} \xi_{\mu, v, \pi}^{\lambda}=\xi_{\lambda, \mu, v \pi} \xi_{\lambda \mu, v, \pi}$, Deutsche Math. 5 (1940), 138-149.

UNIVERSITY OF ILLINOIS,

URBANA, ILLINOIS

University of Illinois at Chicago Circle,

Chicago, Illinois 